\title{
Content-Based Image Retrieval by Query Adaptive Search using Hash Codes
}

\author{
E. Aarthi ${ }^{1}$, S. Kaviya ${ }^{2}$, D. Keerthika ${ }^{3}$, B. Kirithika ${ }^{4}$ \\ UG, Department of CSE, MKCE, Karur, India ${ }^{1,2,3,4}$
}

\begin{abstract}
Visual similarity has been a lively topic of analysis in Scalable image search. Progressive solutions usually use hashing ways to plant high-dimensional image options into playing area, wherever search may be performed in period supported playing distance of compact hash codes. Not like ancient metrics (e.g., Euclidean) that provide continuous distances, the playing distances area unit separate number values. As a consequence, there area unit usually an outsized range of pictures sharing equal playing distances to a question that mostly hurts search results wherever fine-grained ranking is vital. This paper introduces associate approach that allows query-adaptive ranking of the came pictures with equal playing distances to the queries. This can be achieved by first off offline learning bitwise weights of the hash codes for a various set of predefined linguistics thought categories. We have a tendency to formulate the burden education method as a quadratic programming drawback that minimizes intra-class distance whereas conserving inter-class relationship captured by original raw image options. Query-adaptive weights area unit then computed online by evaluating the proximity between a question and also the linguistics thought categories.
\end{abstract}

Keywords: Hash codes, Query-adaptive, Hamming distance.

\section{INTRODUCTION}

The explosion of pictures on the web, there's a powerful have to be compelled to develop techniques for economical and climbable image search. Whereas ancient image search engines heavily accept matter words associated to the pictures, climbable content-based search is receiving increasing attention. Excluding providing higher image search expertise for standard net users, largescale similar image search has conjointly been incontestable to be terribly useful for resolution variety of terribly onerous issues in pc vision and transmission like image categorization. Usually a large-scale image search system consists of 2 key components - an effective image feature illustration Associate in nursing an economical search mechanism. Its average search results depends heavily on the illustration power of image options.

The latter, Associate in Nursing economical search mechanism, is dynamic since existing image options area unit principally of high dimensions and current image databases area unit are vast, on high of that thoroughly inspection a question with each information sample is computationally preventive. During this work we have a tendency to represent pictures mistreatment the popular bag-of-visual- words (Bow) framework wherever native invariant image descriptors area unit extracted and quantity supported a group of visual words. For economical search, the Bow options area unit then embedded into compact hash codes. For this, we have a tendency to think about advanced techniques as well as semi-supervised hashing and linguistics hashing with deep belief networks. In tree-based categorization structures hashing is preferred because it usually requests significantly condensed memory for high-dimensional samples. With the hash codes, image similarity are often expeditiously measured (using logical XOR operations) in fantasy area by playacting distance, Associate in Nursing whole number worth obtained by enumeration the quantity of bits at that the binary values area unit totally different.

\section{DOMAIN INTRODUCTION}

Image processing is processing of images using mathematical operations by using any form of signal processing for which the input is an image, a series of images, or a video, such as a photograph or video frame. The output of image processing may be either an image or a set of characteristics or parameters related to the image. Most image-processing techniques involve treating the image as a two-dimensionalsignal and applying standard signal-processing techniques to it. Images are also processed as three-dimensional signals with the thirddimension being time or the $\mathrm{z}$-axis. Image processing usually refers to digital image processing, but optical and analog image processing also are possible.

\section{LITERATURE SURVEY}

\section{[A]Efficient SkylineComputation in Structured Peer-} to-Peer Systems

This paper, investigate the multidimensional skyline computation problem on a structured peer-to-peer network. In order to achieve low communication cost and quick response time, Mini Max method is utilized to transform high-dimensional data to one-dimensional value and distribute the data in a structured peer-to-peer network called BATON. Thereafter, a progressive algorithm with adaptive filter technique was proposed for efficient skyline 
computation in this environment and summarizes the key principles of algorithm into a query routing protocol with detailed analysis.

\section{[B]Achieving Effective Multi-term Queries for Fast} DHT Information Retrieval

Distributed Hash Tables (DHTs) do not directly support multi-term queries but well-suited for exact match lookups using unique identifiers, and to improves the retrieval effectiveness of such query Related study of query expansion has shown that adding new terms to a query via ad hoc feedback. In the paper, an effective multi-term query processing algorithm for information retrieval in DHT systems. Given the significance of first term in a multi-term query, to the peers containing the first term the query is sent. To enhance the query effectiveness and based on users' behaviours two query expansion mechanisms and an implicit relevance feedback approach are used. Additionally, the query log are recorded and the expansion terms for each query which can accelerate the future queries and improve the query accuracy.

\section{[C]Chord: A Scalable Peer-to-peer Lookup Service for} Internet Applications

An important problem that challenges peer-to-peer applications is to efficiently locate the node that stores a particular data item. This paper describes about Chord, a distributed lookup protocol that addresses this problem. Chord provides provision for just one action given a key, it maps the key onto a node. Data location can be easily executed on top of Chord by relating a key with each data item, and storing the key/data item pair at the node to which the key maps. Chord adjusts efficiently as nodes join and leave the system, and can answer questions even if the system is continuously changing. Results from theoretical analysis, simulations, and experiments shows that Chord is scalable, with communication cost and the state kept by each node scaling logarithmically with the number of Chord nodes.

\section{[D]Evaluating Bag of Visual Words Representations in Scene Classification}

Based on key points take out as noticeable image patches, an image can be described as a bag of visual words" and the representation has been often used in the classification of imagery data. The representation choices weighting of visual words are central to the classification performance but have not been thoroughly studied in existing works.

Specified from the analogy between image representation and the bag-of-words representation of text documents, we can apply techniques extensively used in text categorization, feature selection, including weighting, stop word removal, to make image demonstrations that differ in the dimension, selection, and weighting of visual words. The impression of these representations choices to scene classification is calculated through wide experiments on the TRECVID and PASCAL collections. This study provides a foundation for designing visual-word representations that are likely to produce superior classification performance.

[E]Dynamic content-based image search and retrieval by combining low level features

To access the large collection of images the new techniques are very pivotal. Image mining system is the Content Based Image Retrieval (CBIR) which execute retrieval based on the similarity described in terms of extracted features with more advantages. This paper describes dynamic content based image search and retrieval is conferred, called Hybrid feature extraction method. The noticed contents of an image such as shape, colour and texture are availed in content Based Image Retrieval (CBIR).

To improve the performance and accuracy of retrieved images, the Proposed algorithm associate the advantages of distinct algorithms. The accuracy of colour histogram based matching can be noticeable by using Colour Coherence Vector for successive depuration. The Fourier Descriptors with Fast Fourier transform and Extended Hough transform can support the speed of shape based retrieval.

The Gabor filter has been generally accepted to extract image features, peculiarly texture features. Feature Vector Normalization can be established to make sure that different feature vectors in the similarity measurement process. In this way the colour, shape and texture features are united to provide a good feature set for image retrieval. In addition to this, relevance feedback (RF) scheme is superior to progress the attainment of content based image retrieval (CBIR).

\section{EXISTING SYSTEM}

The existing systems adopt a worldwide feature approach: a picture is diagrammatic as a high dimensional feature vector (e.g., color histogram), and therefore the similarity between files is measured victimisation the gap between 2 feature vectors.

Usually, the feature vectors are indexed by a distributed high-dimensional index or neighbourhood Sensitive Hashing (LSH) over the DHT overlay In distinction to centralized environments, information in $\mathrm{P} 2 \mathrm{P}$ networks is distributed among totally different nodes, so a CBIR formula has to index and hunt for pictures in an exceedingly distributed manner.

\section{DISADVANTAGES}

Even once one will update the hash functions with dynamic information, implementing it over the DHTs is extremely difficult. Because the information is hold on among nodes of corresponding hash ID, a 1-bit amendment of the hash operate output can lead to massive portion of (if not all) information being assigned to a distinct node, inflicting significant network traffic. 


\section{PROPOSED SYSTEM}

In this work we tend to represent pictures victimization the favoured bag-of-visual-words (BoW) framework, wherever native invariant image descriptors (e.g., SIFT) are extracted and quantity supported a collection of visual words it typically needs greatly reduced memory and additionally works higher for high-dimensional samples. With the hash codes, image similarity will be with efficiency measured (using logical XOR operations) in performing house by performing distance, associate degree number worth obtained by investigating the quantity of bits at that the binary values are completely different.

\section{EXPERIMENTAL RESULT}

\section{Modules:}

$>$ Query Image

$>$ Feature Extraction

$>$ Generate the image based on the hash code

$>$ Retrieve the image and generate the graphs

\section{CONCLUSION}

We have given a completely unique framework for queryadaptive image search with hash codes. By harnessing an outsized set of predefined linguistics thought categories, our approach is in a position to predict query-adaptive bitwise weights of hash codes in period, with that search results will be quickly graded by weighted playing distance at finer-grained hash code level. Our findings indicate that the class-specific codes will any improve search performance considerably.

\section{REFERENCES}

[1] Chord: A Scalable Peer-to-peer Lookup Service for Internet Applicationsvol.31, no. 2, pp. 26-34, 1998.

[2] Efficient Skyline Computation in Structured Peer-to-Peer Systems, IEEE Transactions on Knowledge and Data Engineering, vol. , no. , 2008

[3] 1D.Vetrithangam, 2Dr.N.Uma Maheswari 3Dr.R.Venkatesh, Dynamic content-based image search and retrieval by combining low level features , N.UmaMaheswari et al., International Journal of Advanced Engineering Technology E-ISSN 0976-3945 Int J AdvEngg Tech/Vol. VII/Issue II/April-June,2016/898-906 Research Paper

[4] Alexander G. Hauptmann, Chong-Wah Ngo, Evaluating Bag-ofVisual-Words Representations in Scene Classification, no. , 2008

[5] Quanqing Xu1, Heng Tao Shen2, Yafei Dai1, Bin Cui1, and XiaofangZhou2, Achieving Effective Multi-term Queries for Fast DHT Information Retrieval, J. Bailey et al. (Eds.): WISE 2008, LNCS 5175, pp. 20-35, 2008.

[6] S.Saravanan, Arivarasan."An efficien ranked keyword search for effective utilization of outsourced cloud data" Journal of Global Research in Computer Science, Vol4(4), pp:8-12

[7] S Saravanan, V Venkatachalam ," Improving map reduce task scheduling and micro-partitioning mechanism for mobile cloud multimedia services" International Journal of Advanced Intelligence Paradigms, Vol 8(2),pp157- 167,2016.

[8] S Saravanan, V Venkatachalam ," Advance Map Reduce Task Scheduling algorithm using mobile cloud multimedia services architecture" IEEE Digital Explore,pp21-25,2014.
[9] S.Swathi "Preemptive Virtual Machine Scheduling Using CLOUDSIM Tool”, International Journal of Advances in Engineering, 2015, 1(3), 323 -327 ISSN: 2394-9260, pp:323-327.

[10] S Saravanan, V Venkatachalam, S Then Malligai "Optimization of SLA violation in cloud computing using artificial bee colony" 2015 , 1(3), 323 -327 ISSN: 2394-9260, pp:410-414.

[11] S. Saravanan, Vikram R ," Improved Performance Analysis Image Segmentation Based on Cluster Image", Journal of Chemical and Pharmaceutical Sciences,issue 1,2017,pp92-95

[12] S. Saravanan, Vikram R, "Evolutionary Calculations on Gravitational Interactions Method of Global Leader Organize ", Journal of Chemical and Pharmaceutical Sciences,issue 1,2017,pp115-118 